CLINICAL @LERT

\title{
Do those with positive faecal occult blood tests need upper gastrointestinal investigations if no colorectal cancer cause is found?
}

\author{
M H Robinson
}

Should upper gastrointestinal investigation be part of the workup for some or all subjects returning positive Haemoccult faecal occult blood tests as part of a screening programme for the early detection of colorectal cancer?

T hree large randomised controlled trials of faecal occult blood (FOB)

screening using the guaiac based test, Haemoccult, have demonstrated a reduction in mortality from colorectal cancer $^{1-3}$ and two pilot schemes around Coventry and in Eastern Scotland are currently evaluating the feasibility that these research findings can be reproduced in routine practice. Data from these pilots will influence whether a national programme of $\mathrm{FOB}$ screening is implemented in the UK.

The British Society of Gastroenterology recommends that iron deficiency anaemia should be investigated by colonoscopy and gastroscopy. ${ }^{4}$ Occult bleeding is a common cause of iron deficiency anaemia, and Haemoccult detects occult bleeding. It would seem logical to ask whether, as well as colonoscopy, upper gastrointestinal investigation should be carried out in a screened population with a positive FOB test. Rasmussen and Kronborg consider the case for offering upper gastrointestinal investigation to all those returning a positive FOB test or only to those in whom colonoscopy fails to show a colorectal cancer or large $(>1 \mathrm{~cm})$ adenoma. The question is important because the additional cost of a gastroscopy (in all or a majority of test positive subjects) would be a substantial financial burden for the budget of a national screening programme.

The likelihood of a bleeding upper gastrointestinal lesion (cancer or otherwise) resulting in a positive FOB test depends on the rate of blood loss and the type of test used. Faeces contains a mixture of intact haemoglobin, free haem, and porphyrins. Porphyrins will predominate with proximal gastrointestinal bleeding while intact haemoglobin and haem are more evident when the bleeding is distal. All FOB test technologies detect haemoglobin but immunological tests detect none of the degradation products. Guaiac based FOB tests (for example, Haemoccult) also detect haem

Rasmussen M, Kronborg O. Upper gastrointestinal cancer in a population-based screening program with fecal occult blood test for colorectal cancer. Scand J Gastroenterol 2002;37:95-8.

Question: Do those found to be faecal occult blood positive during colorectal cancer screening need investigation of the upper gastrointestinal tract if no cause for blood loss is found in the lower gastrointestinal tract?

Design: Follow up of screened group of a population based randomised trial. Setting: Funen County, Denmark

Subjects: A total of 30967 subjects aged $45-75$ years in 1985, randomised to screening using non-rehydrated Haemoccult tests.

Outcome: Diagnosis of any upper gastrointestinal cancers within two years of screening; cancers were identified from the Funen patient database, cancer registration, and local and national death registration.

Results: A total of 20671 subjects underwent a total of 120165 tests of which 1767 were positive $157 \%$ of 1536 having complete colonic investigations had no colorectal lesion found). Ten had an upper gastrointestinal cancer (five gastric, four pancreatic, one oesophageal) identified within the next two years of which five occurred in those already found to have had a colorectal adenoma or cancer. Of the four cancers in subjects with a "clean" colon, two gastric cancers, both inoperable, were found as a result of investigation of upper gastrointestinal symptoms reported at the time of screening. The positive predictive value of a positive Haemoccult test for upper gastrointestinal cancer was $<1 \%$ and was not significantly greater in those who were also anaemic at the time of screening Conclusion: In the absence of symptoms, investigation of the upper gastrointestinal tract is not justified in those with a positive faecal occult blood test but with no cause found on colonic investigation.

but not haem derived porphyrins while haem-porphyrin assays detect all products of haemoglobin degradation. The ability of the FOB test to detect upper gastrointestinal bleeding has been simulated by faecal testing following oral ingestion of varying amounts of blood. Haem-porphyrin assays are considerably elevated while immunological tests are persistently negative. Haemoccult and other guaiac based tests are only positive at very high haem-porphyrin assay levels. ${ }^{5}$ This suggests that the yield of significant upper gastrointestinal pathology, including cancer, in subjects with a positive Haemoccult test will be low, irrespective of the findings at colonoscopy. This has been the experience in both the Minnesota ${ }^{1}$ and Nottingham trials.

Rasmussen and Kronborg investigated the prevalence of upper gastrointestinal cancer in their cohort of 1767 screened subjects who had returned a positive FOB test. Although none routinely underwent gastroscopy or ultrasound, all were asked about dyspeptic symptoms (18\%) and haemoglobin concentration was checked $(7.1 \%$ had an unspecified anaemia). A diagnosis of upper gastrointestinal cancer in this group was established by cross referencing this patient database with local, regional, and national cancer registries. A total of 10 patients (oesophagus one; stomach five; pancreas four) were found to have had an upper gastrointestinal cancer diagnosed within two years of a positive Haemoccult test. Those with dyspepsia or anaemia were no more likely to have an upper gastrointestinal cancer than those without this symptom or finding. Five of the 10 cases also had a large adenoma or cancer in their colon. On this 
basis it could be argued that upper gastrointestinal investigation should be done in all test positive cases in the same way that dual investigation is recommended for the investigation of iron deficiency anaemia. ${ }^{4}$ However, 115 upper gastrointestinal tests (presumably upper gastrointestinal endoscopy plus ultrasound/computerised tomography) would need to be done for each upper gastrointestinal cancer detected, in contrast with 10 colonoscopies for each colorectal cancer detected. Furthermore, only four of the 10 cases were operable at the time of investigation although this figure may have been higher with earlier diagnosis. A selective policy of upper gastrointestinal investigation (only if colonoscopy was "negative") would have led to only five upper gastrointestinal cancers potentially detected of which only one was operable at the time of presentation. Each of these five cancers would have required 175 sets of investigation for its detection. With neither policy can such a rate of return be justified-as well as having marginal effectiveness, it would clearly add considerably to the cost of the screening.

The biology of upper gastrointestinal tract bleeding and the technology of the FOB test used in all of the large screening trials suggest that upper gastrointestinal investigation of test positives for cancer detection would be ineffective and expensive. The paper of Rasmussen and Kronborg supports this.

\section{Gut 2002;51:466-467}

\section{Author's affiliation}

M H Robinson, Department of Surgery, E Floor, West Block, University Hospital, Queen's

Medical Centre, Clifton Boulevard, Nottingham NG7 2UH, UK;

Mike.Robinson@mail.qmcuh-tr.trent.nhs.uk

\section{REFERENCES}

1 Mandel JS, Bond JH, Church TR, et al. Reducing mortality from colorectal cancer by screening for fecal occult blood. Minnesota Colon Cancer Control Study. N Engl J Med 1993:328: 1365-71.

2 Hardcastle JD, Chamberlain JO, Robinson $\mathrm{MH}$, et al. Randomised controlled trial of faecal occult blood screening for colorectal cancer. Lancet 1996;348:1472-7.

3 Kronborg O, Fenger C, Olsen J, et al. Randomised study of screening for colorectal cancer with faecal occult blood test. Lancet 1996;348: 1477-1.

4 Goddard AF, Mclntyre AS, Scott BB. British Society of Gastroenterology guidelines for the management of iron deficiency anaemia. Gut 2000;46(suppl 3-4):IV1-5.

5 Young GP. Biology of bleeding from neoplasia: implications for occult blood testing. In: Young GP, Saito H, eds. Faecal occult blood tests: current issues and new tests. SmithKline Diagnostics, 1992:10-18.

6 Thomas WM, Hardcastle JD. Role of upper $\mathrm{Gl}$ investigations in a screening study for colorectal neoplasia. Gut 1990;31:1294-7.

\section{Clinical Evidence-Call for contributors}

Clinical Evidence is a regularly updated evidence based journal available world wide both as a paper version and on the internet. Clinical Evidence urgently needs to recruit a number of new contributors. Contributors are health care professionals or epidemiologists with experience in evidence based medicine and the ability to write in a concise and structured way.

We are presently interested in finding contributors with an interest in the following clinical areas:

\section{Angina pectoris Attention deficit hyperactivity disorder Genital warts Hepatitis B}

\section{Hepatitis C \\ HIV \\ Influenza \\ Varicose veins}

\section{Being a contributor involves:}

- Appraising the results of literature searches (performed by our Information Specialists) to identify high quality evidence for inclusion in the journal.

- Writing to a highly structured template (about 1500-3000) words), using evidence from selected studies, within 6-8 weeks of receiving the literature search results.

- Working with Clinical Evidence Editors to ensure that the text meets rigorous epidemiological and style standards.

- Updating the text every eight months to incorporate new evidence.

- Expanding the topic to include new questions once every eight months to incorporate new evidence.

- Expanding the topic to include new questions once every 12-18 months.

If you would like to become a contributor for Clinical Evidence or require more information about what this involves, please send your contact details and a copy of your $\mathrm{CV}$, clearly stating the clinical area you are interested in, to Polly Brown (pbrown@bmigroup.com). 